Contemporary Mathematics

Volume 218, 1998

B $0-8218-0988-1-03001-6$

\title{
A Non-Overlapping Domain Decomposition Method for the Exterior Helmholtz Problem
}

\author{
Armel de La Bourdonnaye, Charbel Farhat, Antonini Macedo, \\ Frédéric Magoulès, and François-Xavier Roux
}

\section{Introduction}

In this paper, we first show that the domain decomposition methods that are usually efficient for solving elliptic problems typically fail when applied to acoustics problems. Next, we present an alternative domain decomposition algorithm that is better suited for the exterior Helmholtz problem. We describe it in a formalism that can use either one or two Lagrange multiplier fields for solving the corresponding interface problem by a Krylov method. In order to improve convergence and ensure scalability with respect the number of subdomains, we propose two complementary preconditioning techniques. The first preconditioner is based on a spectral analysis of the resulting interface operator and targets the high frequency components of the error. The second preconditioner is based on a coarsening technique, employs plane waves, and addresses the low frequency components of the error. Finally, we show numerically that, using both preconditioners, the convergence rate of the proposed domain decomposition method is quasi independent of the number of elements in the mesh, the number of subdomains, and depends only weakly on the wavenumber, which makes this method uniquely suitable for solving large-scale high frequency exterior acoustics problems.

Acoustic wave propagation problems lead to linear systems that become very large in the high frequency regime. Indeed, for most discretization methods, the mesh size $h$ is typically chosen as one tenth of the wavelength in order to ensure a basic approximation of the physical phenomena. For this reason, many iterative solvers have been and continue to be developed for the Helmholtz problem. In this paper, we consider a domain decomposition based iterative algorithm, because of the success encountered by such methods for the solution of elliptic problems, and because they can be easily implemented on parallel computers.

1991 Mathematics Subject Classification. Primary 65N55; Secondary 35J05, 65Y05.

Key words and phrases. Domain decomposition, Lagrange multipliers; exterior Helmholtz problem; Krylov method, preconditioning, coarse grid. 
The problem we are interested in solving arises from the discretization of the Helmholtz equation in a bounded domain $\Omega$ with an outgoing boundary condition on the outside boundary $\Gamma=\partial \Omega$, and can be written as follows

Problem 1 (The exterior Helmholtz problem). Let $f \in L^{2}(\Omega)$ and $f^{s} \in L^{2}(\Gamma)$. Find $u \in H^{1}(\Omega)$ so that

$$
\begin{gathered}
-\triangle u-k^{2} u=f \text { in } \Omega \\
\frac{\partial u}{\partial n}+\alpha u=f^{s} \text { on } \Gamma
\end{gathered}
$$

\section{Domain decomposition for exterior acoustics problems}

2.1. Classical domain decomposition methods for elliptic problems. For elliptic problems, non-overlapping domain decomposition methods are usually preferred. In such methods, one splits the initial domain $\Omega$ into a finite set of $N$ subdomains $\Omega_{i}$ satisfying

$$
\bar{\Omega}=\bigcup_{i=1}^{N} \bar{\Omega}_{i}, \quad \Omega_{i} \cap \Omega_{j}=\emptyset \quad \forall i \neq j
$$

Let $N_{i}=\left\{j, j \neq i, \partial \Omega_{i} \cap \partial \Omega_{j} \neq \emptyset\right\}$, be the set of indices $j$ of the subdomains $\Omega_{j}$ that are neighbors of $\Omega_{i}$. For such problems, most domain decomposition methods require solving the restriction to each subdomain of the global equation with a set of boundary conditions imposed on the subdomain interfaces. For a suitable choice of boundary conditions and constraints on the subdomain interfaces, each local problem is a well-posed one, and the local solutions $u_{i}$ in $\Omega_{i}$ are the restrictions to each subdomain of the global solution in $\Omega$.

In the FETI method (cf. C. Farhat and F.-X. Roux $[\mathbf{9 ,} \mathbf{1 0}]$ ), known also as a dual Schur complement method, the following interface conditions are used on $\Gamma_{i j}=\partial \Omega_{i} \cap \partial \Omega_{j}$

$$
\frac{\partial u_{i}}{\partial n_{i j}}=\lambda_{i j}=-\lambda_{j i}=-\frac{\partial u_{j}}{\partial n_{j i}} \quad \text { on } \Gamma_{i j} \quad u_{i}=u_{j}
$$

Problem 2 (The Dual Schur problem). Let $f \in L^{2}(\Omega)$. Find $u_{i} \in H^{1}\left(\Omega_{i}\right)$ satisfying

$$
-\triangle u_{i}=f_{\mid \Omega_{i}} \text { in } \Omega_{i}
$$

$$
\frac{\partial u_{i}}{\partial n_{i j}}=\lambda_{i j} \text { on } \Gamma_{i j} \quad \forall j \in N_{i}
$$

under the constraint

$$
u_{i}-u_{j}=0 \text { on } \Gamma_{i j} \quad \forall j \in N_{i}
$$

On the other hand, the primal Schur complement method (cf. P. le Tallec [12]) uses a Dirichlet boundary condition on the subdomain interfaces, which ensures the continuity of the solution through these interfaces with the constraint

$$
\frac{\partial u_{i}}{\partial n_{i j}}+\frac{\partial u_{j}}{\partial n_{j i}}=0 \text { sur } \Gamma_{i j} \quad \forall j \in N_{i}
$$

For such a method and in each subdomain, on has 
Problem 3 (Primal Schur problem). Let $f \in L^{2}(\Omega)$. Find $u_{i} \in H^{1}\left(\Omega_{i}\right)$ satisfying

$$
\begin{gathered}
-\triangle u_{i}=f_{\mid \Omega_{i}} \text { in } \Omega_{i} \\
u_{i}=p_{i j}\left(=p_{j i}\right) \text { on } \Gamma_{i j} \quad \forall j \in N_{i}
\end{gathered}
$$

under the given constraint

$$
\frac{\partial u_{i}}{\partial n_{i j}}+\frac{\partial u_{j}}{\partial n_{j i}}=0 \text { on } \Gamma_{i j} \quad \forall j \in N_{i}
$$

REMARK 4. The local solutions obtained by any of the above methods satisfy the following continuity equations

$$
\begin{gathered}
u_{i}-u_{j}=0 \text { on } \Gamma_{i j} \quad \forall j \in N_{i} \\
\frac{\partial u_{i}}{\partial n_{i j}}+\frac{\partial u_{j}}{\partial n_{j i}}=0 \text { on } \Gamma_{i j} \quad \forall j \in N_{i}
\end{gathered}
$$

These two equalities ensure that the function $u$ which is equal to $u_{i}$ in each subdomain $\Omega_{i}$ is the solution of the global problem in $H^{1}(\Omega)$.

If any of the two domain decomposition methods presented above is used for solving the Helmholtz problem, the associated local problems can become ill-posed when the wavenumber $k$ of the given global problem corresponds to a resonant frequency of the subdomain Laplace operator. It follows that the interface boundary conditions characteristic of domain decomposition methods for strongly elliptic problems cannot be used for the Helmholtz equation (see also [1]).

In $[\mathbf{3}]$ and $[\mathbf{4}]$, B. Desprès presents a domain decomposition method for the Helmholtz problem where the local subproblems are well-posed, but where a simple (and rather inefficient) relaxation-like iterative method is employed for solving the resulting interface problem. In this paper, we formulate the interface problem in terms of Lagrange multipliers, and develop a scalable preconditioned Krylov method for solving it.

2.2. A new domain decomposition method for the Helmholtz problem. One way to generate well-posed local problems consists in moving the spectrum of the operator associated to the Helmholtz equation in each subdomain into the complex plane. For example, one can replace the standard Dirichlet or Neumann boundary conditions on the subdomain interfaces by the Robin boundary conditions $\Omega_{i}$ and $\Omega_{j}, \forall j \in N_{i}$, can be written as

$$
\begin{array}{ll}
\frac{\partial u_{i}}{\partial n}+i k u_{i}=\lambda_{i j} \text { on } \Gamma_{i j} \quad \forall j \in N_{i} \\
\frac{\partial u_{j}}{\partial n}+i k u_{j}=\lambda_{j i} \text { on } \Gamma_{i j} \quad \forall j \in N_{i}
\end{array}
$$

where $\lambda_{i j}-\lambda_{j i}=0$ and $n$ is either $n_{i j}$ or $n_{j i}$. The constraint on the subdomain interfaces is determined so that local solutions $u_{i}$ and $u_{j}$ satisfy the continuity relations (12) and (13). Hence, this constraint can be formulated as

$$
\left[\frac{\partial u}{\partial n}-i k u\right]=0 \text { on } \Gamma_{i j} \quad \forall j \in N_{i}
$$


where the brackets $[\cdots]$ denote the jump of the enclosed quantity through the interface $\Gamma_{i j}$ between two subdomains. It follows that the problem to be solved in each subdomain is

Problem 5. Let $f \in L^{2}(\Omega)$ and $f^{s} \in L^{2}(\Gamma)$. Find $u_{i} \in H^{1}\left(\Omega_{i}\right)$ satisfying

$$
\begin{gathered}
-\triangle u_{i}-k^{2} u_{i}=f_{\mid \Omega_{i}} \text { in } \Omega_{i} \\
\frac{\partial u_{i}}{\partial n}+i k u_{i}=\lambda_{i j} \text { on } \Gamma_{i j} \quad \forall j \in N_{i} \\
\frac{\partial u_{i}}{\partial n}+\alpha u_{i}=f^{s} \text { on } \Gamma \cap \partial \Omega_{i}
\end{gathered}
$$

with the constraint

$$
\left[\frac{\partial u}{\partial n}-i k u\right]=0 \text { on } \Gamma_{i j} \quad \forall j \in N_{i}
$$

Strictly speaking, the above method is correct only for a slice-wise or a checkerboard-like decomposition of $\Omega$. For arbitrary mesh partitions, the above method is not guaranteed to generate well-posed local problems, unless additional precaution is taken [7]. Indeed, the variational formulation of problem (5) is

$$
\begin{gathered}
\forall v_{i} \in H^{1}\left(\Omega_{i}\right), \quad \int_{\Omega_{i}} \nabla u_{i} \nabla v_{i}-k^{2} \int_{\Omega_{i}} u_{i} v_{i}+\alpha \int_{\Gamma \cap \partial \Omega_{i}} u_{i} v_{i} \\
=\int_{\Omega_{i}} f_{i} v_{i}+\int_{\Gamma \cap \partial \Omega_{i}} f_{i}^{s} v_{i}+\sum_{j \in N_{i}} \int_{\Gamma_{i j}} \frac{\partial u_{i}}{\partial n_{i j}} v_{i}
\end{gathered}
$$

Substituting in the above equation the normal derivative with the expression derived from formula (18) leads to the following Lax-Milgram lemma bilinear form

$$
\int_{\Omega_{i}} \nabla u_{i} \nabla v_{i}-k^{2} \int_{\Omega_{i}} u_{i} v_{i}+\alpha \int_{\Gamma \cap \partial \Omega_{i}} u_{i} v_{i}-i k \sum_{j \in N_{i}}(-1)^{\delta_{n_{i j}}^{n}} \int_{\Gamma_{i j}} u_{i} v_{i}
$$

where $\delta_{n_{i j}}^{n}$ is equal to 1 if $n$ is the outgoing normal unit vector to $\Omega_{i}$, and is equal to 0 otherwise. The $H^{1}$-Ellipticity of the functional is then not satisfied for some partitions of the domain $\Omega$ and the problem becomes locally ill-posed. However, as shown in [7] and [5], coloring techniques can be used to extend the domain decomposition method proposed above to arbitrary mesh partitions while ensuring well-posed local problems. Alternatively, one can address general partitions of $\Omega$ by relaxing the equality $\lambda_{i j}-\lambda_{j i}=0$ and introducing independent Lagrange multipliers $\lambda_{i j}$ and $\lambda_{j i}$. In that case, in each subdomain $\Omega_{i}, n$ is chosen as the outgoing unit normal vector, and the global constraint is modified so that in each subdomain the following problem is solved

Problem 6. Let $f \in L^{2}(\Omega)$ and $f^{s} \in L^{2}(\Gamma)$. Find $u_{i} \in H^{1}\left(\Omega_{i}\right)$ so that

$$
\begin{gathered}
-\triangle u_{i}-k^{2} u_{i}=f_{\mid \Omega_{i}} \text { in } \Omega_{i} \\
\frac{\partial u_{i}}{\partial n_{i j}}+i k u_{i}=\lambda_{i j} \text { on } \Gamma_{i j} \quad \forall j \in N_{i} \\
\frac{\partial u_{i}}{\partial n_{i}}+\alpha u_{i}=f^{s} \text { on } \Gamma \cap \partial \Omega_{i}
\end{gathered}
$$


with the double constraint

$$
\left[\frac{\partial u}{\partial n_{i j}}+i k u\right]=0 \quad \text { and } \quad\left[\frac{\partial u}{\partial n_{j i}}+i k u\right]=0 \text { on } \Gamma_{i j} \quad \forall j \in N_{i}
$$

Note that the above double constraint can be derived from linear combinations of the continuity relations (12) and (13). Indeed, one has

$$
\begin{aligned}
& (26 a)=(13)+i k(12) \\
& (26 b)=(13)-i k(12)
\end{aligned}
$$

Proceeding this way, we note that by inverting (26a) and (26b), the following alternative double constraint is obtained

$$
\left[\frac{\partial u}{\partial n_{i j}}-i k u\right]=0 \quad \text { and } \quad\left[\frac{\partial u}{\partial n_{j i}}-i k u\right]=0 \text { on } \Gamma_{i j} \quad \forall j \in N_{i}
$$

Nevertheless, we prefer formula (26) because it leads to a linear system of interface equations whose spectrum and conditioning properties are preferable for iterative solution methods (see $[\mathbf{8}]$ ).

REMARK 7. The domain decomposition method presented here is correctly defined for a strictly positive wavenumber $k$, but becomes singular when $k$ goes to 0 . In the latter case, the double constraint becomes

$$
\left[\frac{\partial u}{\partial n_{i j}}\right]=0 \quad \text { and } \quad\left[\frac{\partial u}{\partial n_{j i}}\right]=0 \text { on } \Gamma_{i j} \quad \forall j \in N_{i}
$$

and relation (12) coupling the traces of the local solutions on the interfaces is never satisfied. It follows that the method presented in this section cannot be used for solving the Laplace equation.

Notation 8. In the following, we denote by $Q_{i j}$ the operator

$$
Q_{i j}: \frac{\partial u_{i}}{\partial n_{i j}}+i k u_{i} \quad \mapsto \frac{\partial u_{i}}{\partial n_{i j}}-i k u_{i}
$$

It is shown in [2] that $Q_{i j}$ is a unitary operator. Furthermore, its spectrum has an accumulation point at 1 . Numerically, the density around this point is inversely proportional to the wavenumber $k$.

\section{Variational formulation of the proposed domain decomposition method}

3.1. A Lagrange multiplier formulation. In order to analyze the domain decomposition method presented in this paper, we begin by rewriting the Helmholtz problem (1) as a hybrid problem with two Lagrange multiplier fields on the subdomain interfaces. Following [11] for the Laplace equation, we write the variational formulation of our target problem as follows. Let $f \in L^{2}(\Omega)$ and $f^{s} \in L^{2}(\Gamma)$, find $u \in H^{1}(\Omega)$ so that

$$
\forall v \in H^{1}(\Omega), \quad \int_{\Omega} \nabla u \nabla v-k^{2} \int_{\Omega} u v+\alpha \int_{\Gamma} u v=\int_{\Omega} f v+\int_{\Gamma} f^{s} v
$$

Next, we consider a decomposition of $\Omega$ into $N$ subdomains

$$
\bar{\Omega}=\bigcup_{i=1}^{N} \bar{\Omega}_{i}, \quad \Omega_{i} \cap \Omega_{j}=\emptyset \quad \forall i \neq j
$$


and rewrite the variational formulation (30) as follows. Let $f \in L^{2}(\Omega)$ and $f^{s} \in$ $L^{2}(\Gamma)$. Find $u \in H^{1}(\Omega)$ so that

$$
\begin{gathered}
\forall v \in H^{1}(\Omega), \quad \sum_{i=1}^{N} \int_{\Omega_{i}} \nabla\left(u_{\mid \Omega_{i}}\right) \nabla\left(v_{\mid \Omega_{i}}\right)-k^{2} \int_{\Omega_{i}}\left(u_{\mid \Omega_{i}}\right)\left(v_{\mid \Omega_{i}}\right)+\alpha \int_{\Gamma \cap \partial \Omega_{i}}\left(u_{\mid \Omega_{i}}\right)\left(v_{\mid \Omega_{i}}\right) \\
=\sum_{i=1}^{N} \int_{\Omega_{i}}\left(f_{\mid \Omega_{i}}\right)\left(v_{\mid \Omega_{i}}\right)+\int_{\Gamma \cap \partial \Omega_{i}}\left(f_{\mid \Omega_{i}}^{s}\right)\left(v_{\mid \Omega_{i}}\right)
\end{gathered}
$$

where $\left(v_{\mid \Omega_{i}}\right)$ is the restriction of $v$ to $\Omega_{i}$. Instead of looking for a function $u$ defined in $\Omega$, it is easier to look for an $N$-uple $u^{*}=\left(u_{1}, \cdots, u_{N}\right)$ belonging to a space $V^{*}$ spanned by these restrictions.

$$
V^{*}=\left\{v^{*}=\left(v_{1}, \cdots, v_{N}\right), \exists v \in H^{1}(\Omega), \forall i, 1 \leq i \leq N, v_{i}=v_{\mid \Omega_{i}}\right\}
$$

The space $V^{*}$ can be written in terms of the space $X^{*}$ which is the product of the spaces $X_{i}$, defined by

$$
X_{i}=\left\{v_{i} \in H^{1}\left(\Omega_{i}\right)\right\}, \quad X^{*}=\prod_{i=1}^{N} X_{i}
$$

as follows

$$
V^{*}=\left\{v^{*}=\left(v_{1}, \cdots, v_{N}\right) \in X^{*}, \forall i, 1 \leq i \leq N, \forall j \in N_{i}, v_{i \mid \Gamma_{i j}}=v_{j \mid \Gamma_{i j}}\right\}
$$

where $v_{i \mid \Gamma_{i j}}$ is the trace on the interface $\Gamma_{i j}$ of the function $v_{i}$. The constraint on the subdomain interfaces can be relaxed by introducing a double Lagrange multiplier $\left(\lambda_{i j}, \lambda_{j i}\right)$ in the equation as presented in the previous section. This Lagrange multiplier belongs to the space $M$ included in $\prod_{1 \leq i \leq N} \prod_{j \in N_{i}} H^{-\frac{1}{2}}\left(\Gamma_{i j}\right)$. The initial problem is thus equivalent to the following constrained problem

Problem 9. Let $f \in L^{2}(\Omega)$ and $f^{s} \in L^{2}(\Gamma)$, find $u \in V^{*}$ so that :

$$
\begin{gathered}
\forall v \in V^{*} \quad \sum_{i=1}^{N} \int_{\Omega_{i}} \nabla u_{i} \nabla v_{i}-k^{2} \int_{\Omega_{i}} u_{i} v_{i}+\alpha \int_{\Gamma \cap \partial \Omega_{i}} u_{i} v_{i} \\
=\sum_{i=1}^{N} \int_{\Omega_{i}} f_{i} v_{i}+\int_{\Gamma \cap \partial \Omega_{i}} f_{i}^{s} v_{i}
\end{gathered}
$$

and, with the notation introduced above, to the hybrid problem

Problem 10. Let $f \in L^{2}(\Omega)$ and $f^{s} \in L^{2}(\Gamma)$, find $\left(u^{*}, \lambda\right) \in X^{*} \times M$ so that

$$
\begin{gathered}
\forall v^{*} \in X^{*}, \quad \sum_{i=1}^{N} \int_{\Omega_{i}} \nabla u_{i} \nabla v_{i}-k^{2} \int_{\Omega_{i}} u_{i} v_{i}+\alpha \int_{\Gamma \cap \partial \Omega_{i}} u_{i} v_{i} \\
+\sum_{j \in N_{i}} \int_{\Gamma_{i j}}\left(-\lambda_{i j}+i k u_{i \mid \Gamma_{i j}}\right) v_{i \mid \Gamma_{i j}}=\sum_{i=1}^{N} \int_{\Omega_{i}} f_{i} v_{i}+\int_{\Gamma \cap \partial \Omega_{i}} f_{i}^{s} v_{i} \\
\forall v \in X^{*}, \quad \sum_{i=1}^{N} \sum_{j \in N_{i}} \int_{\Gamma_{i j}}\left(\lambda_{i j}+\lambda_{j i}-2 i k u_{i \mid \Gamma_{i j}}\right) v_{i \mid \Gamma_{i j}}=0
\end{gathered}
$$




$$
\sum_{i=1}^{N} \sum_{j \in N_{i}} \int_{\Gamma_{i j}}\left(\lambda_{i j}+\lambda_{j i}-2 i k u_{j \mid \Gamma_{i j}}\right) v_{i \mid \Gamma_{i j}}=0
$$

In the sequel, we discretize problem (10) for conforming meshes using the method of $[\mathbf{1 0}]$.

3.2. Discretization of the governing equations. Discretizing the hybrid formulation derived in the previous section leads to

$$
A_{i} u_{i}=f_{i}+\sum_{j \in N_{i}} B_{i j}^{t} \lambda_{i j}
$$

where the matrix $A_{i}$ results from the discretization of the bilinear form

$$
\int_{\Omega_{i}} \nabla u_{i} \nabla v_{i}-k^{2} \int_{\Omega_{i}} u_{i} v_{i}+\alpha \int_{\Gamma \cup \partial \Omega_{i}} u_{i} v_{i}+i k \sum_{j \in N_{i}} \int_{\Gamma_{i j}} u_{i \mid \Gamma_{i j}} v_{i \mid \Gamma_{i j}}
$$

and $B_{i j}$ represents the operator

$$
\left(B_{i j} u_{i}, \lambda_{i j}\right)=\left(u_{i}, B_{i j}^{t} \lambda_{i j}\right)=\int_{\Gamma_{i j}} \lambda_{i j} u_{i \mid \Gamma_{i j}}
$$

The question that arises first is that of the choice of the space of discretization for the constraint variables $\lambda_{i j}$ and $\lambda_{j i}$. But, as our problem is not exactly a hybrid one, there is no way to fulfil the Ladyzhenskaya-Babuska-Brezzi condition uniformly. As argued in the next remark, this is not a problem in our case, and following the analysis presented in [10], we can choose for the operator $B_{i j}$ the restriction to the interface of the operator $R_{i j}$. Such an approach corresponds to choosing for the constraint fields the space of the Dirac masses that are centered on the nodes of the subdomain interfaces. Let us recall that this choice ensures the equality of the discrete fields on the interface for the Laplace equation. Hence, if one defines $M_{i j}$ as the mass matrix on the subdomain interfaces

$$
\left(M_{i j} R_{i j} u_{i}, R_{j i} u_{j}\right)=\left(R_{j i}^{t} M_{i j} R_{i j} u_{i}, u_{j}\right)=\int_{\Gamma_{i j}} u_{i \mid \Gamma_{i j}} u_{j \mid \Gamma_{i j}}
$$

one can discretize the first constraint of problem (10)

$$
\int_{\Gamma_{i j}}\left(\lambda_{i j}+\lambda_{j i}-2 i k u_{i \mid \Gamma_{i j}}\right) u_{j \mid \Gamma_{i j}}=0
$$

as

$$
\left(\lambda_{i j}+\lambda_{j i}-2 i k M_{i j} R_{i j} u_{i}, R_{j i} u_{j}\right)=0
$$

which can be rewritten as

$$
R_{j i}^{t}\left(\lambda_{i j}+\lambda_{j i}-2 i k M_{i j} R_{i j} u_{i}\right)=0
$$

Taking into account that $R_{i j}=\left[\begin{array}{ll}0 & I\end{array}\right]$, on can deduce

$$
\lambda_{i j}+\lambda_{j i}-2 i k M_{i j} R_{i j} u_{i}=0
$$

Applying the same treatment to the second constraint, one finally obtains

$$
\sum_{i=1}^{N} \sum_{j \in N_{i}} \lambda_{i j}+\lambda_{j i}-2 i k M_{i j} R_{i j} u_{i}=0
$$




$$
\sum_{i=1}^{N} \sum_{j \in N_{i}} \lambda_{i j}+\lambda_{j i}-2 i k M_{j i} R_{j i} u_{j}=0
$$

REMARK 11. Adding equations (47) and (48), one obtains the following system of equations

$$
\sum_{i=1}^{N} \sum_{j \in N_{i}}-2 i k M_{i j}\left(R_{i j} u_{i}-R_{j i} u_{j}\right)=0
$$

and thus

$$
\left(R_{i j} u_{i}-R_{j i} u_{j}\right)=0 \quad \forall i \text { and } j \in N_{i}
$$

In other words, the discrete fields $u_{i}$ are continuous across the subdomain interfaces. Hence, one can assemble the local equations and show that the local discrete solutions in each subset $\Omega_{i}$ are indeed the restrictions of the discrete solutions of the global problem since there is no approximation in the way the constraints are satisfied.

Substituting $u_{i}$ and $u_{j}$ obtained in equation (39) into equation (47) and equation (48), leads to the following interface problem

$$
\begin{aligned}
& \sum_{i=1}^{N} \sum_{j \in N_{i}} \lambda_{i j}+\lambda_{j i}-2 i k M_{i j} R_{i j} A_{i}^{-1} R_{i j}^{t} \lambda_{i j}=2 i k M_{i j} R_{i j} A_{i}^{-1} f_{i} \\
& \sum_{i=1}^{N} \sum_{j \in N_{i}} \lambda_{i j}+\lambda_{j i}-2 i k M_{j i} R_{j i} A_{j}^{-1} R_{j i}^{t} \lambda_{j i}=2 i k M_{j i} R_{j i} A_{j}^{-1} f_{j}
\end{aligned}
$$

Denoting by $\lambda$ the double Lagrange multiplier $\left(\lambda_{i j}, \lambda_{j i}\right)$ defined on all the interfaces, the previous system can be written as

$$
D \lambda=b
$$

where $D$ is a dense, complex, regular, unsymmetric and non hermitian matrix. This matrix is not explicitely known, and assembling it is computationally inefficient. However, given that $D$ is the sum over the subdomains of local matrices, its product by a vector needs only local data (see Section 2.3.2). For these reasons, an iterative method is the most suitable method for solving the linear system $D \lambda=b$.

\subsection{Iterative solution of the the hybrid problem.}

3.3.1. The Generalized Conjugated Residuals algorithm. Among all iterative methods, the Generalized Conjugated Residuals algorithm (GCR) is perhaps the most efficient for solving the linear system $D \lambda=b$ where $D$ is a complex matrix. This algorithm minimizes $\|D \lambda-b\|^{2}$ on the Krylov spaces $K_{p+1}=\left\{g_{o}, D g_{0}, \cdots\right.$, $\left.D^{p} g_{0}\right\}$ with growing dimension $p$.

Knowing at iteration $p$ the approximate solution $\lambda^{p}$, the residual $g^{p}=D \lambda^{p}-b$, the normalized descent direction vectors $\left\{w^{0}, \cdots, w^{p}\right\}$ and their product by matrix $D,\left\{D w^{0}, \cdots, D w^{p}\right\}$, the iteration $p+1 \mathrm{~d}$ of the GCR algorithm goes as follows

- Compute the optimal descent coefficient

$$
\rho^{p}=-\left(g^{p}, \overline{D w^{p}}\right)
$$


- Update the solution and the residual

$$
\begin{gathered}
\lambda^{p+1}=\lambda^{p}+\rho^{p} w^{p} \\
g^{p+1}=g^{p}+\rho^{p} D w^{p}
\end{gathered}
$$

- Compute the product of $D w^{p}$ by matrix $D$

$$
D^{2} w^{p}
$$

- Determine the new descent direction by orthogonalizing with respect to the $D^{\star} D$ inner product the vector $D w^{p}$ and all the previously computed search directions

$$
\begin{gathered}
\gamma_{j}^{p}=-\left(D^{2} w^{p}, \overline{D w^{j}}\right) \quad \forall j=0, \cdots, p \\
w^{p+1}=D w^{p}+\sum_{j=0}^{p} \gamma_{j}^{p} w^{j}
\end{gathered}
$$

- Compute $D w^{p+1}$

$$
D w^{p+1}=D^{2} w^{p}+\sum_{j=0}^{p} \gamma_{j}^{p} D w^{j}
$$

- Normalize $w^{p+1}$

$$
w^{p+1}=\frac{w^{p+1}}{\sqrt{\left(D w^{p+1}, \overline{D w^{p+1}}\right)}} \quad D w^{p+1}=\frac{D w^{p+1}}{\sqrt{\left(D w^{p+1}, \overline{D w^{p+1}}\right)}}
$$

where $(w, v)$ represents the scalar product of the vectors $w$ and $v$ in $L^{2}(\Gamma \times \Gamma)$, and $\bar{w}$ the complex conjugate of the vector $w$. Using the properties of orthogonality of the different vectors, one can show that this algorithm converges with a number of iterations that is smaller or equal to the dimension of matrix $D$.

3.3.2. Cost and implementation issues. The main part of the computations is associated with the matrix-vector product, for which one has to solve a Helmholtz problem with radiation conditions at each iteration, and in each subdomain. The remainder of the computation consists of scalar products and linear combinations of vectors.

As stated previously, the product of a vector by matrix $D$ needs only data that is local to each processor. This product is performed by first using matrices $A_{i}^{-1}, M_{i j}$ and $R_{i j}$ which are local to the subdomains, and then assembling the result over all the subdomains.

The fact that $A_{i}$ is a symmetric matrix allows us to use a Crout factorization so that the products by matrix $A_{i}^{-1}$ can be obtained by forward and backward substitutions. Since matrix $R_{i j}$ is a restriction matrix, it does not need be stored. Furthermore, the rank of matrix $M_{i j}$ is equal to the number of degrees of freedom on the interface between two subdomains. The use of a direct local solver, and the properties of the operator on the interface ensure that the proposed domain decomposition method has good convergence properties and is more robust than other iterative methods. Also note that because only local matrices are factored, this method is more economical than direct ones.

From the implementation viewpoint, the assembly part requires exchanging messages containing one-dimensional arrays defined on the interfaces such as descent directions, or Lagrange multipliers. Therefore, the amount of data exchanged 
Neumann B.C.

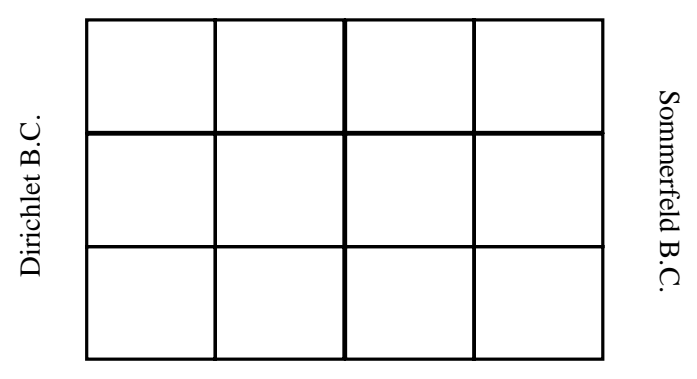

Neumann B.C.

Figure 1. Problem definition
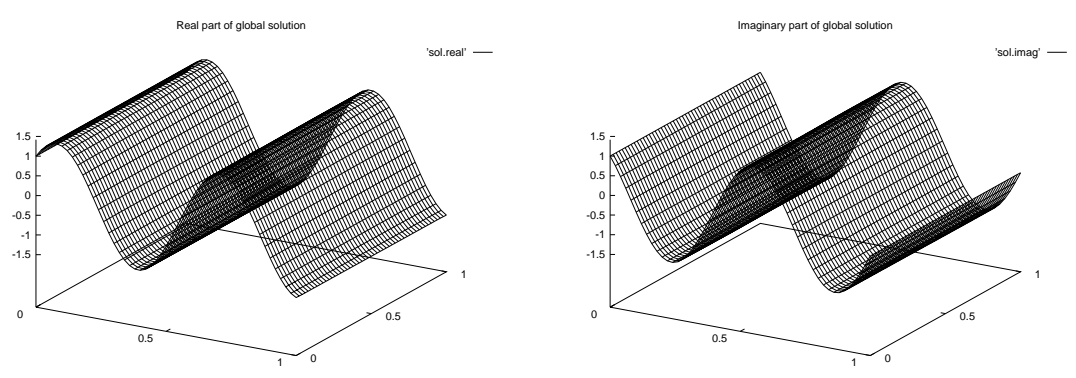

FIgURE 2. Real and imaginary parts of the exact solution

is small compared to the amount of computations performed. In other terms, the product of a vector by matrix $D$ consists in local forward and backward substitutions followed by data exchanges between processors, and therefore the proposed method is easily parallelizable on any multiprocessor.

\section{Numerical scalability analysis}

Here, we perform a set of numerical experiments to assess the convergence of the proposed method for an increasing problem size, and/or an increasing number of subdomains, and/or an increasing wavenumber. More specifically, we consider a two-dimensional rectangular waveguide problem with a uniform source located on the west side, and reflecting boundaries at the north and south sides. The exact solution of this sharp problem is a plane wave traveling from west to east. The domain $\Omega$ is discretized by finite elements and partitioned in a number of subdomains. Homogeneous Neumann boundary conditions are applied on the north and south sides of the domain, a non-homogeneous Dirichlet condition is applied on its west side, and an absorbing condition on its east side.

The geometry of the domain and the real and imaginary parts of the exact solution are shown on Fig. 1 and Fig. 2.

First, we investigate the dependence of the convergence of the proposed method on the mesh size (Fig. 3). For this purpose, we fix the wavenumber $k$ and the number of subdomains and consider a series of mesh sizes $h, h / 2, h / 3, \ldots$. The 


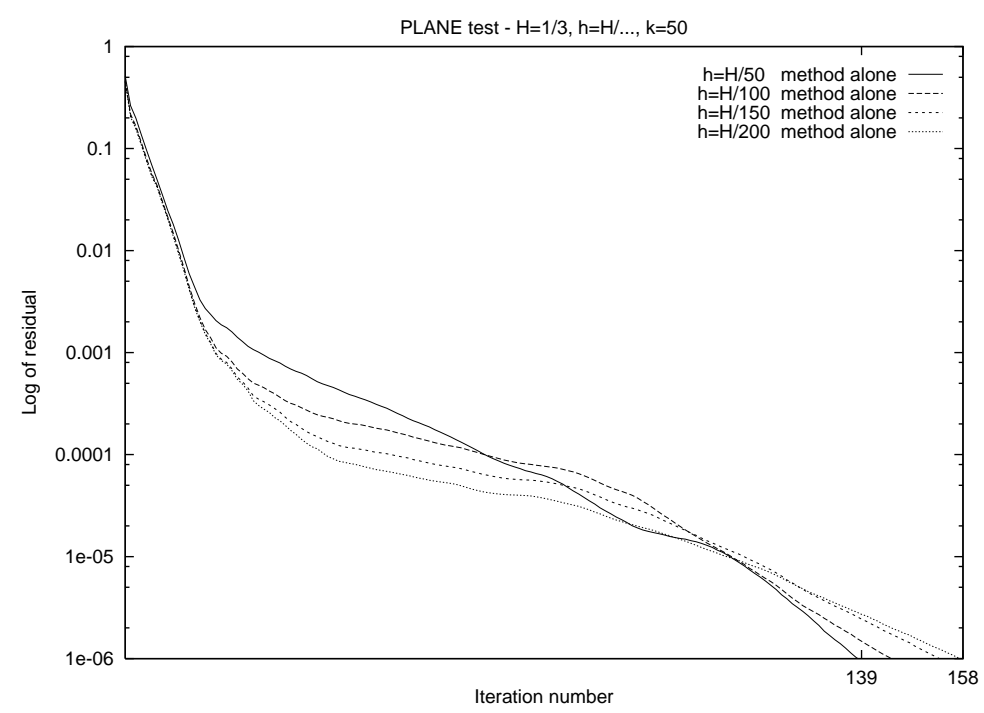

FiguRE 3. Effect on convergence of the mesh size

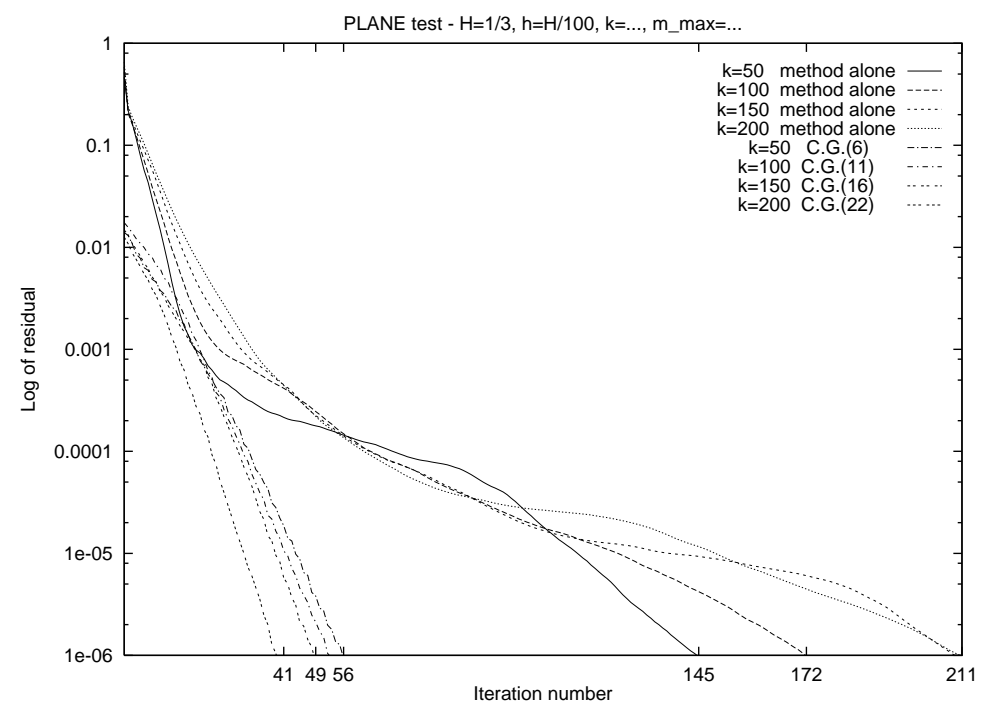

FiguRE 4. Effect on convergence of the wavenumber

results reported in Figure 3 show that the convergence of the proposed domain decomposition method is only weakly dependent on the mesh size $h$, which is quite impressive given that no preconditioner has yet been explicitly introduced. This corroborates the fact that operator $Q_{i j}$ is unitary.

The next figure depicts the varation of the convergence of the method with respect to the wavenumber for a fixed mesh size and a fixed number of subdomains (Figure 4). The results reported in Figure 4 reveal a sublinear dependence on the wavenumber $k$. Practically, this indicates that when the frequency of the problem is increased, the convergence of the method deteriorates. 


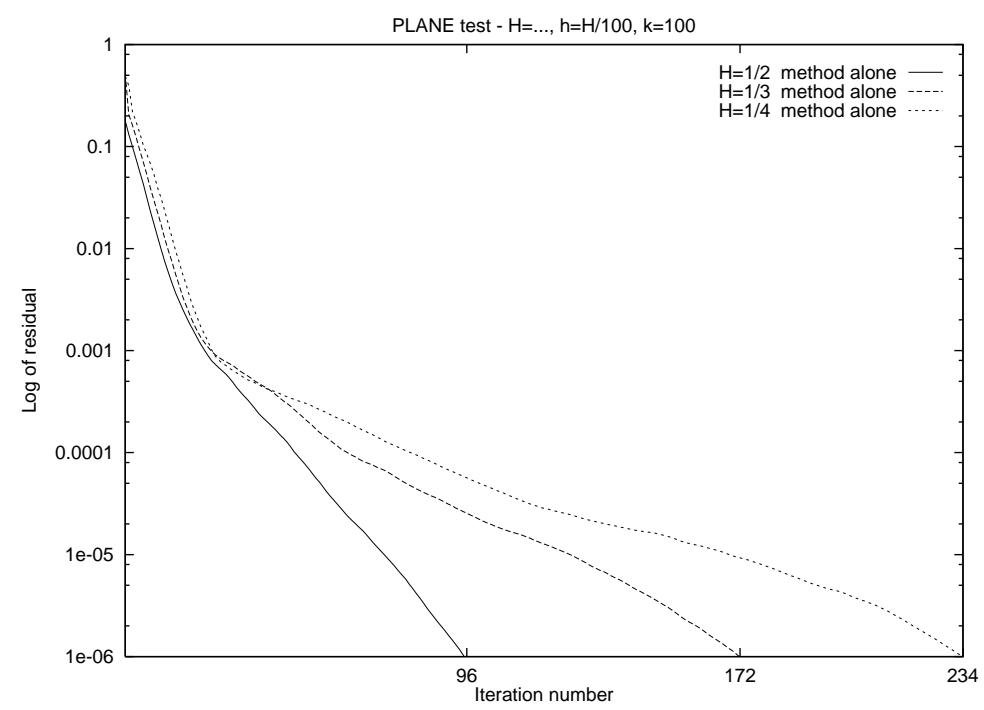

FIgURE 5. Effect on convergence of the number of subdomains

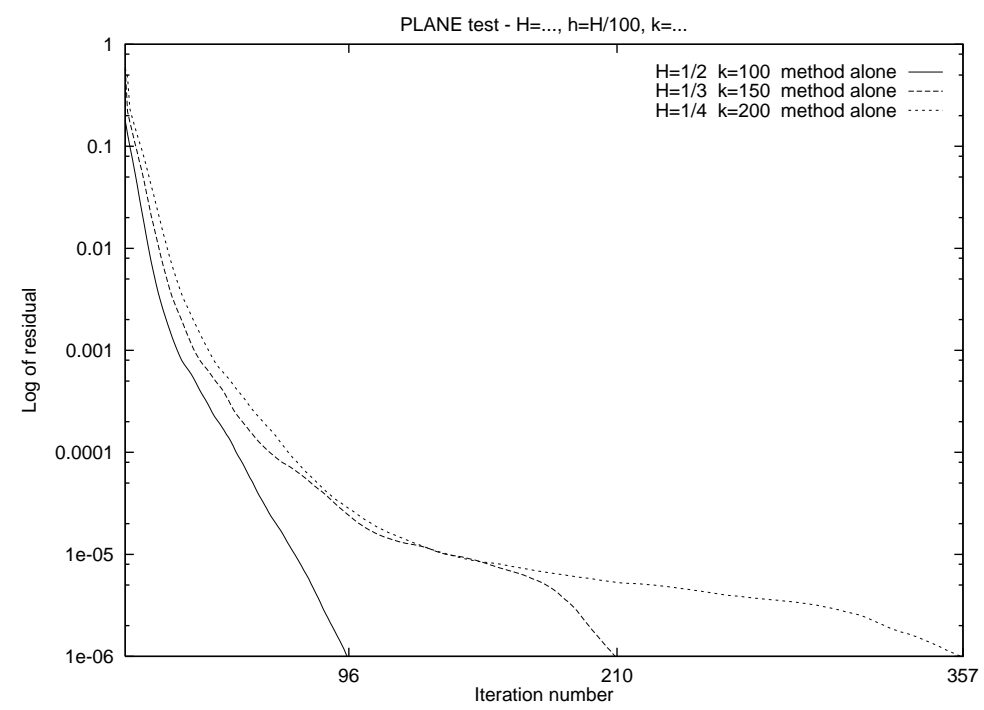

Figure 6. Convergence with a fixed number of wavelengths per subdomain

In order to study the effect of the number of subdomains, we fix the number of degrees of freedom in the local meshes and the wavenumber, and increase the number of subdomains. The corresponding results (Figure 5) show a linear dependence with respect to the number of subdomains. The last study (Figure 6) shows the effect on convergence of a simultaneous variation of the wavenumber and the number of subdomains where $k H$ is kept constant, $H$ being the mean diameter of a subdomain. The results clearly show a linear dependence of convergence on $k H$.

In summary, the method as presented so far seems to scale with the problem size (mesh size), but not with the wavenumber and/or the number of subdomains. 


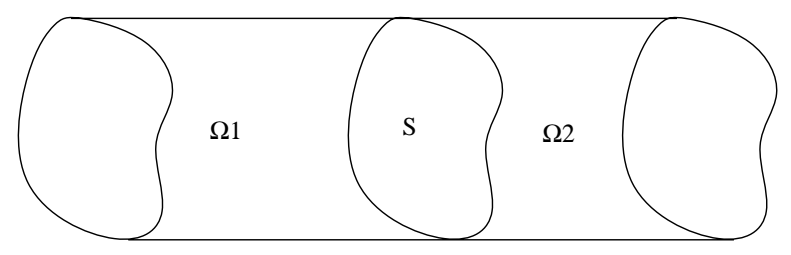

Figure 7. Geometry of the model problem

Therefore, our next objective is to develop preconditioning techniques for ensuring scalability with respect to the number of subdomains, and improving convergence in the high frequency regime.

\section{Preconditioning techniques}

5.1. Definitions. Here, we restrict our analysis to the following canonical problem (cf Figure 7).

Let $S$ be a bounded set of $\mathbb{R}^{2}$ with a regular surface. Domain $\Omega$ under consideration is $S \times[0, L]$. We use two subdomains $\Omega_{1}$ and $\Omega_{2}$ defined by

$$
\begin{aligned}
& \Omega_{1}=S \times\left[0, L_{1}\right] \\
& \Omega_{2}=S \times\left[L_{1}, L_{1}+L_{2}\right]
\end{aligned}
$$

with $0<L_{1}, 0<L_{2}$ and $L_{1}+L_{2}=L$. The sectional variables are denoted by $x, y$, the fiber variable is denoted by $z$. We will also use $(x, y, z)=(Y, z)=X$. We denote by $\Gamma_{I}=S \times L_{1}$ the interface between $\Omega_{1}$ and $\Omega_{2}$.

The interesting point about this problem is that it separates the variables and facilitates the explanation of some features of the method.

Using the previously introduced notation, the matrix $D$ of the condensed problem can be written as

$$
D=\left[\begin{array}{cc}
I & Q_{2} \\
Q_{1} & I
\end{array}\right]
$$

where $Q_{1}$ (resp. $Q_{2}$ ) is a discrete form of the unitary operator introduced in the notation (29), associated with subdomain $\Omega_{1}$ (resp. $\Omega_{2}$ ). The spectrum of matrix $D$ spreads on the unit circle of the complex plane centered in 1 and has two accumulation points : one in 0 and the other in 2 (cf. Fig. 8).

REMARK 12. If the existence of areas of accumulation of eigenvalues generally accelerates the convergence of Krylov-like methods, it is nonetheless clear that the accumulation point located in 0 deteriorates the conditioning of matrix $D$.

REMARK 13. Increasing the frequency for a given mesh has two effects. First, it diminishes the density around the accumulation points and increases the dispersion of the spectrum. Second, the numerical accumulation points move away from 0 and 2. These two properties have contradictory effects on the convergence speed and explain the various crossings of the convergence curves. 


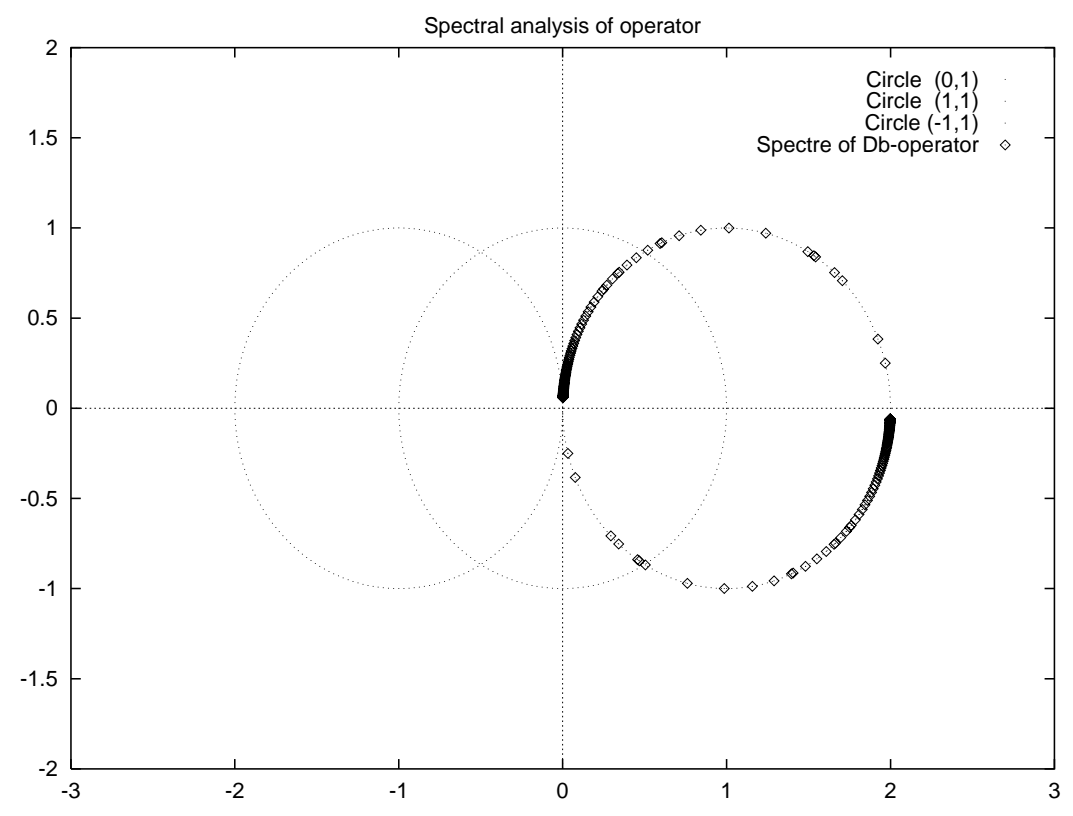

FiguRE 8. Spectral analysis

5.2. Spectral analysis. When considering our canonical problem, the solutions of the Helmholtz problem in $\Omega_{1}$ and $\Omega_{2}$ may be decomposed as the sums of

$$
\psi_{n}(z) \phi_{n}(Y)
$$

where $\phi_{n}$ are the eigenfunctions of the Laplace operator on $S$ denoted by $\Delta_{S}$. Hence, we have

$$
\Delta_{S} \phi_{n}=-\left(\lambda_{n}\right)^{2} \phi_{n}
$$

Thus, the functions $\psi_{n}$ in each of the two subdomains satisfy the ordinary differential equation

$$
\psi_{n}^{\prime \prime}+\left[k^{2}-\left(\lambda_{n}\right)^{2}\right] \psi_{n}=0 .
$$

Two cases must be distinguished

- $k^{2}>\left(\lambda_{n}\right)^{2}$ : this is the propagative case. We denote $k_{n}^{\prime}=\sqrt{k^{2}-\left(\lambda_{n}\right)^{2}}$ and we have $\psi_{n}(z)=a e^{i k_{n}^{\prime} z}+b e^{i k_{n}^{\prime} z}$.

- $k^{2}>\left(\lambda_{n}\right)^{2}$ : this is the case of a vanishing wave. We denote $k_{n}^{\prime}=$ $\sqrt{-k^{2}+\left(\lambda_{n}\right)^{2}}$ and we have $\psi_{n}(z)=a e^{k_{n}^{\prime} z}+b e^{k_{n}^{\prime} z}$.

In each case, $a$ and $b$ are determined from the boundary conditions at $z=0$ or $z=L$.

Let us interpret physically the above two cases. The first case corresponds to a wave which oscillates slowly on the interface and propagates through the subdomains. The second case corresponds to a wave which oscillates so rapidly on the interface that it cannot propagate in the subdomains. In order to speed up convergence, we develop two complementary preconditioners that target the two different cases. The high frequency phenomena on the interface - which corresponds to vanishing waves in the subdomains and hence local waves around the interfaces 
— will be filtered by a local preconditioner. We will construct the local preconditioner as an approximate inverse of the hermitian part of $D$. The low frequency phenomena - which corresponds to waves propagating in all the subdomains will be filtered by a global preconditioner that will be referred to in the sequel as the Coarse Grid preconditioner. This coarse grid preconditioner is a projection on the space orthogonal to a set of functions which are defined on the interfaces. Here, these functions are chosen as low frequency plane waves defined locally on each interface $\Gamma_{i j}$.

\subsection{The local preconditioner.}

5.3.1. The principle. Here, our goal is to replace the solution of the linear system $D \lambda=b$ by the solution of the system $M D \lambda=M b$, where matrix $M$ is an approximate inverse of matrix $\left(D+D^{\star}\right) / 2$. We denote by $n$ the vector normal to $\Gamma_{I}$ and pointing towards the increasing $z$. Thus,

$$
\begin{aligned}
& Q_{1}: \frac{\partial u^{1}}{\partial n}+i k u^{1} \mapsto \frac{\partial u^{1}}{\partial n}-i k u^{1} \\
& Q_{2}:-\frac{\partial u^{2}}{\partial n}+i k u^{2} \quad \mapsto \quad-\frac{\partial u^{2}}{\partial n}-i k u^{2}
\end{aligned}
$$

Let us represent $Q_{1}$ (resp. $Q_{2}$ ) in the basis of the functions $\phi_{n}(Y)$.

If $\left.u^{1}\right|_{\Gamma_{I}}=\phi_{n}(Y)$, then, in $\Omega_{1}$,

$$
u^{1}=\frac{\psi_{n}(z)}{\psi_{n}\left(L_{1}\right)} \phi_{n}(Y)
$$

and

$$
\partial_{n} u^{1}=\frac{\psi_{n}^{\prime}\left(L_{1}\right)}{\psi_{n}\left(L_{1}\right)} \phi_{n}(Y)
$$

Hence,

$$
Q_{1} \quad: \quad \phi_{n}(Y) \mapsto \frac{\psi_{n}^{1^{\prime}}\left(L_{1}\right)-i k \psi_{n}^{1}\left(L_{1}\right)}{\psi_{n}^{1^{\prime}}\left(L_{1}\right)+i k \psi_{n}^{1}\left(L_{1}\right)} \phi_{n}(Y) .
$$

Similarly,

$$
Q_{2} \quad: \quad \phi_{n}(Y) \mapsto \frac{-\psi_{n}^{2^{\prime}}\left(L_{2}\right)-i k \psi_{n}^{2}\left(L_{2}\right)}{-\psi_{n}^{2}\left(L_{2}\right)+i k \psi_{n}^{2}\left(L_{2}\right)} \phi_{n}(Y) .
$$

In the case of Dirichlet boundary conditions on the two faces of the cylinder, one has

- $\psi_{n}^{1}(z)=\sin \left(k_{n}^{\prime} z\right)$ in the propagative case,

- $\psi_{n}^{1}(z)=\operatorname{sh}\left(k_{n}^{\prime} z\right)$ in the vanishing case,

- $\psi_{n}^{2}(z)=\sin \left(k_{n}^{\prime}(z-L)\right)$ in the propagative case,

- $\psi_{n}^{1}(z)=\operatorname{sh}\left(k_{n}^{\prime}(z-L)\right)$ in the vanishing case.

Hence,

- $\frac{\psi_{n}^{1 \prime}}{\psi_{n}^{1}}\left(L_{1}\right)=k_{n}^{\prime} \operatorname{cotg}\left(k_{n}^{\prime} L_{1}\right)$ (prop. case),

- $\frac{\psi_{n}^{1^{\prime}}}{\psi_{n}^{1}}\left(L_{1}\right)=k_{n}^{\prime} \operatorname{coth}\left(k_{n}^{\prime} L_{1}\right)$ (van. case),

- $\frac{\psi_{n}^{2^{\prime}}}{\psi_{n}^{2}}\left(L_{2}\right)=-k_{n}^{\prime} \operatorname{cotg}\left(k_{n}^{\prime} L_{2}\right)$ (prop. case), 
- $\frac{\psi_{n}^{2 \prime}}{\psi_{n}^{2}}\left(L_{2}\right)=-k_{n}^{\prime} \operatorname{coth}\left(k_{n}^{\prime} L_{2}\right)$ (van. case).

It follows that, in the propagative case,

$$
\begin{aligned}
Q_{1}: \phi_{n} & \longrightarrow \frac{k_{n}^{\prime} \operatorname{cotg}\left(k_{n}^{\prime} L_{1}\right)-i k}{k_{n}^{\prime} \operatorname{cotg}\left(k_{n}^{\prime} L_{1}\right)+i k} \phi_{n} \\
Q_{2}: \phi_{n} & \longrightarrow \frac{k_{n}^{\prime} \operatorname{cotg}\left(k_{n}^{\prime} L_{2}\right)-i k}{k_{n}^{\prime} \operatorname{cotg}\left(k_{n}^{\prime} L_{2}\right)+i k} \phi_{n}
\end{aligned}
$$

For the vanishing case, one has to turn the cotangent functions into hyperbolic cotangent functions.

Let us denote by $\lambda_{n}^{1}$ and $\lambda_{n}^{2}$ the eigenvalues of $Q_{1}$ and $Q_{2}$ that have appeared above. In the $\phi_{n}$ basis, operator $D$ can be written as

$$
D=\left[\begin{array}{cc}
1 & \lambda_{n}^{2} \\
\lambda_{n}^{1} & 1
\end{array}\right]
$$

and its hermitian part, denoted by $H D$, is

$$
H D=\left[\begin{array}{cc}
1 & \left(\lambda_{n}^{2}+\bar{\lambda}_{n}^{1}\right) / 2 \\
\left(\lambda_{n}^{1}+\bar{\lambda}_{n}^{2}\right) / 2 & 1
\end{array}\right] .
$$

To filter the vanishing modes, that is, the modes having a spatial frequency on the interface that is greater than the wavenumber of the problem, we are going to look for an approximate inverse of the hermitian part of matrix $D$ in the limit $\lambda_{n}>>k$.

In this limit, we have $: \operatorname{coth}\left(k_{n}^{\prime} L i\right)=1+\mathcal{O}\left(1 / \lambda_{n}^{p}\right), \forall p$. Hence,

$$
\lambda_{n}^{1}=\frac{\left(\lambda_{n}\right)^{2}-2 i k \sqrt{\left(\lambda_{n}\right)^{2}-k^{2}}-2 k^{2}}{\left(\lambda_{n}\right)^{2}}
$$

and,

$$
\lambda_{n}^{2}=\frac{\left(\lambda_{n}\right)^{2}-2 i k \sqrt{\left(\lambda_{n}\right)^{2}-k^{2}}-2 k^{2}}{\left(\lambda_{n}\right)^{2}}
$$

One can deduce

$$
\left(\lambda_{n}^{1}+\bar{\lambda}_{n}^{2}\right) / 2=1-2 \frac{k^{2}}{\left(\lambda_{n}\right)^{2}}+\mathcal{O}\left(1 / \lambda_{n}^{p}\right), \forall p
$$

Similarly,

$$
\left(\bar{\lambda}_{n}^{1}+\lambda_{n}^{2}\right) / 2=1-2 \frac{k^{2}}{\left(\lambda_{n}\right)^{2}}+\mathcal{O}\left(1 / \lambda_{n}^{p}\right), \forall p .
$$

The inverse of $H D$ can be written as

$$
(H D)^{-1}=\left[\begin{array}{cc}
1 & -\left(\lambda_{n}^{2}+\bar{\lambda}_{n}^{1}\right) / 2 \\
-\left(\lambda_{n}^{1}+\bar{\lambda}_{n}^{2}\right) / 2 & 1
\end{array}\right] \frac{1}{1-\left|\frac{\lambda_{n}^{2}+\bar{\lambda}_{n}^{1}}{2}\right|^{2}} .
$$

which simplifies to

$$
(H D)^{-1}=(1 / 4)\left[\begin{array}{cc}
1+\frac{\left(\lambda_{n}\right)^{2}}{k^{2}} & 1-\frac{\left(\lambda_{n}\right)^{2}}{k^{2}} \\
1-\frac{\left(\lambda_{n}\right)^{2}}{k^{2}} & 1+\frac{\left(\lambda_{n}\right)^{2}}{k^{2}}
\end{array}\right]+\mathcal{O}\left(1 / \lambda_{n}^{2}\right) .
$$


Since the functions $\phi_{n}$ are eigenfunctions of $\Delta_{S}$ with $-\left(\lambda_{n}\right)^{2}$ as eigenvalues,

$$
(H D)^{-1}=(1 / 4)\left[\begin{array}{cc}
1-\frac{\Delta_{S}}{k^{2}} & 1+\frac{\Delta_{S}}{k^{2}} \\
1+\frac{\Delta_{S}}{k^{2}} & 1-\frac{\Delta_{S}}{k^{2}}
\end{array}\right]+L
$$

where $L$ is a continuous operator from Sobolev space $H^{s}$ into $H^{s+2}$. We will use the first part of the formula defining $(H D)^{-1}$ as a local preconditioner.

The goal of this local preconditioner is to filter the eigenmodes associated with the eigenvalues that are close to zero. But, as a drawback, it also changes the behavior of the other modes, and principally the low frequency ones.

Hence, we will have to correct this drawback by designing a preconditioner that is associated with the propagative modes in order to achieve a good convergence. This will be done by our coarse grid preconditioner that we present and discuss later in this paper.

5.3.2. The Preconditioned Generalized Conjugated Residual algorithm. In the case where a preconditioner $M$ of matrix $D$ is known, on can use a modified Generalized Conjugated Residual algorithm to solve the linear system $D \lambda=b$. In this algorithm, the successive descent direction vectors are built in order to create a $D^{*} D$ orthogonal basis of the successive Krylov spaces

$$
K_{p+1}=\left\{M g_{0}, M D M g_{0}, \cdots,(M D)^{p} M g_{0}\right\} .
$$

The algorithm is now presented in details.

- Initialization

$$
\lambda_{0}, \quad g_{0}=D \lambda_{0}-b, \quad w^{0}=M g_{0}
$$

- Computation of the product of vector $w^{0}$ by matrix $D$ then normalization

$$
D w^{0} /\left(D w^{0}, \overline{D w^{0}}\right)^{1 / 2}
$$

- Iteration $p+1$ of Preconditioned generalized conjugated residuals algorithm for $p \geq 0$

- Determination of the optimal descent cœefficient

$$
\rho^{p}=-\left(g^{p}, \overline{D w^{p}}\right)
$$

- Update of the solution and its residual

$$
\begin{gathered}
\lambda^{p+1}=\lambda^{p}+\rho^{p} w^{p} \\
g^{p+1}=g^{p}+\rho^{p} D w^{p}
\end{gathered}
$$

- Computation of the product of vector $D w^{p}$ by matrix $M$

$$
M D w^{p}
$$

- Computation of the product of $M D w^{p}$ by matrix $D$

$$
D M D w^{p}
$$

- Determination of the new descent direction by orthogonalizing for $D^{\star} D$ vector $M D w^{p}$ with respect to the previously computed directions

$$
\gamma_{j}^{p}=-\left(D M D w^{p}, \overline{D w^{j}}\right) \quad \forall j=0, \cdots, p
$$




$$
w^{p+1}=M D w^{p}+\sum_{j=0}^{p} \gamma_{j}^{p} w^{j}
$$

- Determination of vector $D w^{p+1}$

$$
D w^{p+1}=D M D w^{p}+\sum_{j=0}^{p} \gamma_{j}^{p} D w^{j}
$$

- Normalization of the new descent direction

$$
w^{p+1}=\frac{w^{p+1}}{\sqrt{\left(D w^{p+1}, \overline{D w^{p+1}}\right)}} \quad D w^{p+1}=\frac{D w^{p+1}}{\sqrt{\left(D w^{p+1}, \overline{D w^{p+1}}\right)}}
$$

5.3.3. Cost issues. The local preconditioner described above requires only a matrix vector multiplication on the subdomain interfaces, and therefore is economical and parallelizable. It is reminiscent of the "lunmped" preconditioner for elasticity problems $[\mathbf{1 0}]$.

\subsection{The coarse grid preconditioner.}

5.4.1. The principle. The goal of this method which was first introduced by Farhat, Chen, and Mandel in [5] for time-dependent elasticity problems is to build an $n+1$ dimensional space $W$ for a space $V$ called Coarse Grid, and then to perform the iterations of GCR in a space orthogonal to $W$. With a good choice of the basis functions of the coarse grid, we aim at a better convergence of the algorithm, since it starts with the initial knowledge of $n+1$ descent directions. From what has been shown above, we choose for $V$, the space spanned by $\left\{v_{0}, \cdots, v_{m}\right\}$ where the $v_{i}$ are low frequency functions on an interface. This space will filter the eigenmodes associated with propagative phenomena.

More details on the theory of the coarse grid preconditioner can be found in [6]. In this paper, we implement the coarse grid preconditioner by means of reconjugations within the GCR algorithm. Of course it is not the unique way to do this and we could have followed a method using the definition of matrix operators as presented in [5] for instance, but the results would not have been changed.

In order to use the projected GCR algorithm, one has to build a basis $W=$ $\left\{w_{0}, \cdots, w_{n}\right\}$ that is $D^{*} D$ orthonormal from basis $V$.As matrix $D$ is regular, if vectors $v_{i}$ are linearly independent, it will be the same for vectors $w_{i}$ and therefore $m=n$. We want to construct $W$ so that

$$
w_{i}=\sum_{j=0}^{m} h_{j i} v_{j} \quad \text { and } \quad\left(D w_{i}, \overline{D w_{j}}\right)=0 \quad \forall i \neq j
$$

In matrix notation we have

$$
W=V H \quad \text { such that } \quad(D W, \overline{D W})=I
$$

This approach produces the same effect as a $Q R$ factorization of basis $V$. Let us consider a Cholesky decomposition $L L^{*}$ of matrix $(D V)^{*}(D V)$. Then the equality (94) becomes

$$
(D V H)^{*}(D V H)=H^{*} L L^{*} H=I
$$

By identifying factors, we deduce : $H=L^{-*}$. Hence, the computation of the basis $W$ simply amounts to forward substitutions in the system $W L^{*}=V$. Once all the vectors of $W$ are computed, we can apply the Projected GCR algorithm described next. 
5.4.2. The projected GCR algorithm. When a $D^{*} D$ orthonormal basis $\left\{w^{0}, \cdots\right.$, $\left.w^{n}\right\}$ is known, it is possible to use a modified GCR, where the modification amounts to building the successive descent directions by reconjugating them with vectors $\left\{w^{0}, \cdots, w^{n}\right\}$. The successive steps of the algorithm are

- Initialization

$$
\tilde{\lambda_{0}}, \quad \tilde{g_{0}}=D \tilde{\lambda_{0}}-b
$$

- Re-initialization by reconjugation

$$
\begin{gathered}
\rho_{j}=\left(g^{0}, \overline{D w^{j}}\right) \quad \forall j=0, \cdots, n \\
\lambda_{0}=\tilde{\lambda_{0}}+\sum_{j=0}^{n} \rho_{j} w^{j}, \quad g_{0}=\tilde{g_{0}}+\sum_{j=0}^{n} \rho_{j} D w^{j}
\end{gathered}
$$

- Computation of the product of vector $g_{0}$ by matrix $D$

$$
D g_{0}
$$

- Computation of the first descent direction

$$
\begin{gathered}
\gamma_{j}=-\left(g_{0}, \overline{D w^{j}}\right) \quad \forall j=0, \cdots, n \\
w^{n+1}=g_{0}+\sum_{j=0}^{n} \gamma_{j} w^{j}
\end{gathered}
$$

- Determination of the quantity $D w^{n+1}$

$$
D w^{n+1}=D g_{0}+\sum_{j=0}^{n} \gamma_{j} D w^{j}
$$

- Normalization of the first descent direction

$$
w^{n+1}=\frac{w^{n+1}}{\sqrt{\left(D w^{n+1}, \overline{D w^{n+1}}\right)}} \quad D w^{n+1}=\frac{D w^{n+1}}{\sqrt{\left(D w^{n+1}, \overline{D w^{n+1}}\right)}}
$$

- Iteration $p+1$ of the projected GCR for $p \geq n+1$

- Determination of the optimal descent coefficient

$$
\rho^{p}=-\left(g^{p}, \overline{D w^{p}}\right)
$$

- Update of the solution and the residual

$$
\begin{gathered}
\lambda^{p+1}=\lambda^{p}+\rho^{p} w^{p} \\
g^{p+1}=g^{p}+\rho^{p} D w^{p}
\end{gathered}
$$

- Computation of the product of vector $D w^{p}$ by matrix $D$

$$
D^{2} w^{p}
$$

- Determination of the new descent direction by orthogonalizing for $D^{\star} D$ vector $M D w^{p}$ with respect to the previously computed directions

$$
\gamma_{j}^{p}=-\left(D^{2} w^{p}, \overline{D w^{j}}\right) \quad \forall j=0, \cdots, p
$$




$$
w^{p+1}=D w^{p}+\sum_{j=0}^{p} \gamma_{j}^{p} w^{j}
$$

- Determination of vector $D w^{p+1}$

$$
D w^{p+1}=D^{2} w^{p}+\sum_{j=0}^{p} \gamma_{j}^{p} D w^{j}
$$

- Normalization of the descent direction vector

$$
w^{p+1}=\frac{w^{p+1}}{\sqrt{\left(D w^{p+1}, \overline{D w^{p+1}}\right)}} \quad D w^{p+1}=\frac{D w^{p+1}}{\sqrt{\left(D w^{p+1}, \overline{D w^{p+1}}\right)}}
$$

5.4.3. Cost issues. Here again, the major part of the computation cost resides in the matrix-vector product. The reconjugations are just scalar products and linear combinations of vectors. It follows that the computation of basis $W=$ $\left\{w_{0}, \cdots, w_{n}\right\}$, obtained by $D^{*} D$ orthonormalization of basis $V$ represents an important amount of computation. It would be as expensive as the computation of the $n+1$ first directions of GCR, if basis $V$ were not defined locally, interface by interface. The choice made for basis $V$ and the fact that the Lagrange multipliers are defined on the double of the global interface restrict the computation of the product of a vector $v_{i}$ by matrix $D$ to a product by the local matrix $A_{i}$, which is a forward-backward substitution, and to exchanges of data between neighbor subdomains. The cost to build the basis $W$ is associated with that of performing in each subdomain $\Omega_{i}$ as many forward-backward substitutions as the number of interfaces of the subdomain, and this for each coarse function defined on an interface. On a parallel processor, if the number of coarse grid functions per interface remains constant, an increase of the number of subdomains would have no effect on the local computational cost of basis $W$. The cost of the basis functions of the coarse grid is thus small compared to the cost of the computation of the $n+1$ first directions of the GCR algorithm.

In the following section, we present the convergence curves when the preconditioners proposed here are used. These curves show that the performance of the proposed domain decomposition method equipped with the preconditioners described here exhibits a low dependency on the wavenumber and on the number of subdomains.

\section{Performance of the preconditioned domain decomposition method}

Here, we assess the impact of the preconditioners presented in the previous sections on the convergence of the proposed domain decomposition method. For this purpose, we employ the same test problem as that introduced in Section 4.

In a first step we investigate the influence of the number of coarse grid basis functions on the number of iterations (Figure 9). As stated before, the coarse grid preconditioner aims at filtering the phenomena associated with low frequencies on the interfaces. If we choose a number of coarse grid basis functions that is too small compared to $m_{\max }=k \pi / L$, some low frequency modes will be damped but the others will slow down the method ( $\mathrm{L}$ is the length of the interface). If we increase the number of basis functions per interface to exceed $m_{\max }$, the coarse grid will affect the middle and high frequency oscillations on the interfaces. In other words, the global preconditioner will interfere with the local one. Hence, it seems more 


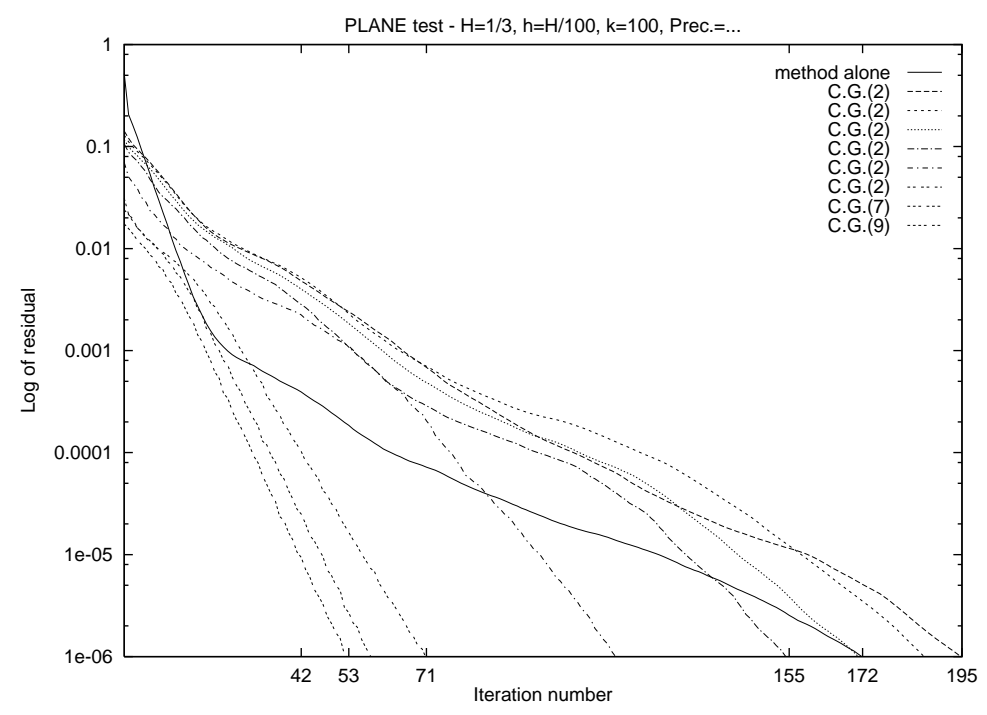

Figure 9. Effect of the size of the coarse problem

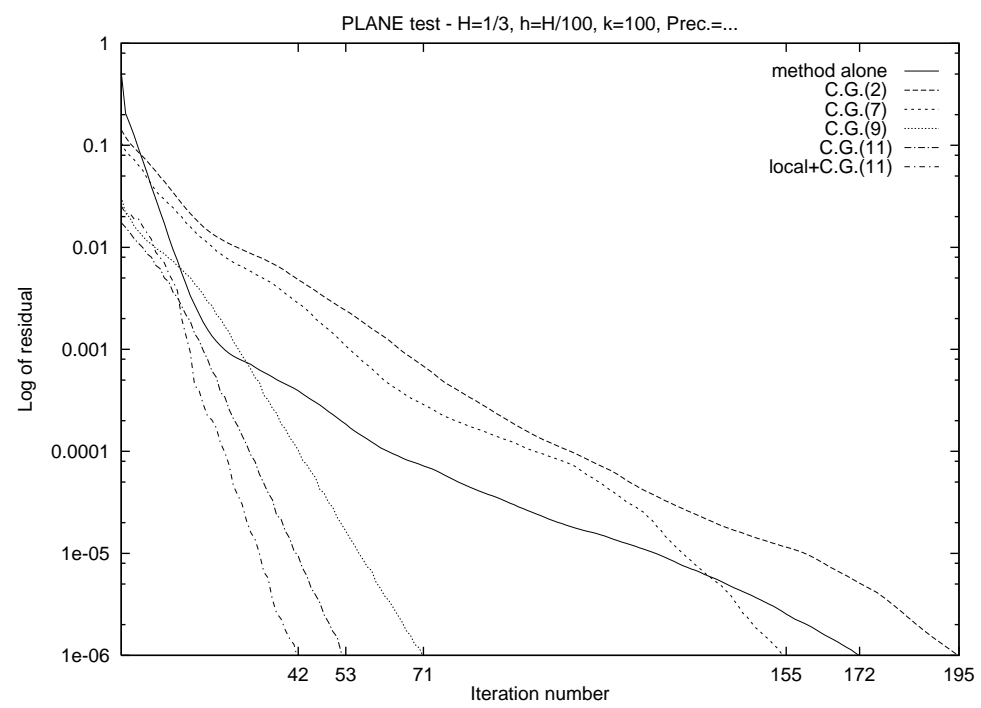

FiguRE 10. Effect of the local preconditioner

sensible to use the coarse grid preconditioner only for low frequencies and to use the local preconditioner for high frequency phenomena. This strategy is also justified by the fact that the local preconditioner increases the granularity of the method without adding reconjugations at each iteration.

In Figure 10, one can see that the local preconditioner accelerates convergence once the low frequency modes have been filtered.

In Section 4, we have exhibited the weak dependency of our method on the mesh size. Here, we show in Figure 11 that this dependency is even weaker when the two preconditioners are used. 


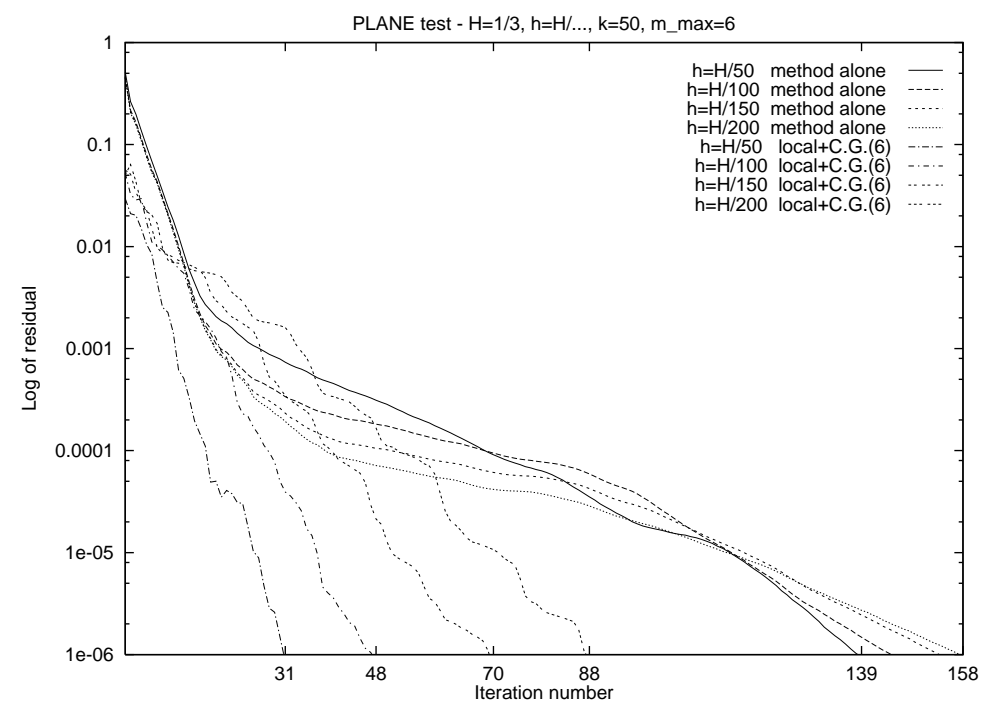

FiguRE 11. Optimal convergence results

Next, we fix the size of the local meshes and the geometry of the global problem and focus on the value of parameter $m_{\max }$, where $m_{\max }$ denotes the largest integer satisfying $m \pi<k H$ where $H$ is the length of a subdomain interface. In other words, all the functions $\phi_{m}(Y)$ with $m>m_{\max }$ correspond to vanishing modes that do not propagate on a long range. We consider a given frequency and a given number of subdomains $N$. If $N$ is increased, the mean diameter $H$ of the subdomains is reduced, and so is $m_{\max }$. The total number of basis functions of the coarse grid space remains unchanged, but, locally the number of basis functions per subdomain decreases; hence, the computational cost is reduced. Figure 12 shows that the coarse grid preconditioner achieves a weak dependence of the method on the the number of subdomains.

Next, we stress that the number of coarse grid functions must vary with the wavenumber $k$. Indeed, when the frequency is increased, one has to proportionally increase the number $m_{\max }$ in order to filter all the propagative modes. Figure 13 shows that, with an appropriate variation of the number of these functions per interface, it is possible to increase the frequency with only a small variation in the convergence histories. If the wavelength diminishes proportionally to the size of the subdomain, i.e. so that the product $k H$ remains constant, one can see that the number $m_{\max }$ remains constant. In other terms, in that case one does not have to increase the number of coarse grid functions for each subdomain interface boundary (see Figure 14). This property is important for realistic exterior acoustics problems.

In summary, the results reported herein show that the performance of the proposed domain decomposition method equipped with the proposed local and global preconditioners is weakly dependent on the mesh size, the subdomain size, and the frequency of the problem, which makes this method uniquely efficient at solving high frequency exterior acoustics problems. 


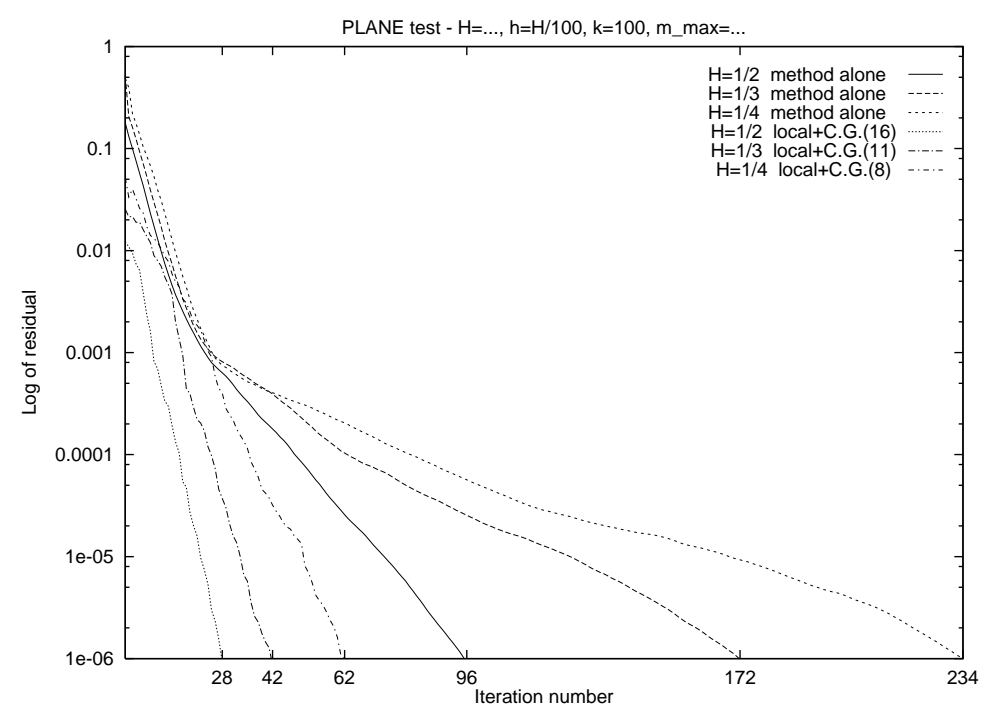

FIGURE 12. Effect of the coarse grid preconditioner

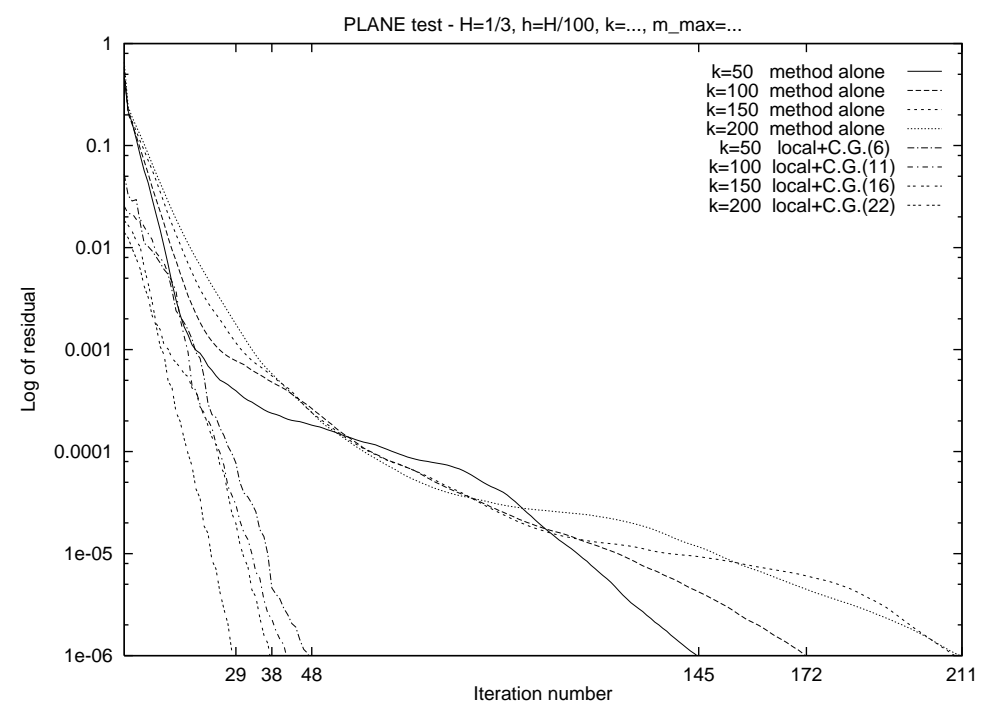

FiguRE 13. Performance results for the high frequency regime

\section{Conclusions}

In this paper, we have presented a Lagrange multiplier based domain decomposition method for solving iteratively large-scale systems of equations arising from the finite element discretization of high-frequency exterior Helmholtz problems. The proposed method relies on three key ideas: (1) the elimination of local resonance via the stabilization of each subdomain operator by a complex interface mass matrix associated with intersubdomain radiation conditions, (2) the use of a carefully constructed local preconditioner for filtering high frequency errors and accelerating convergence in the presence of fine meshes, and (3) the use of a global 


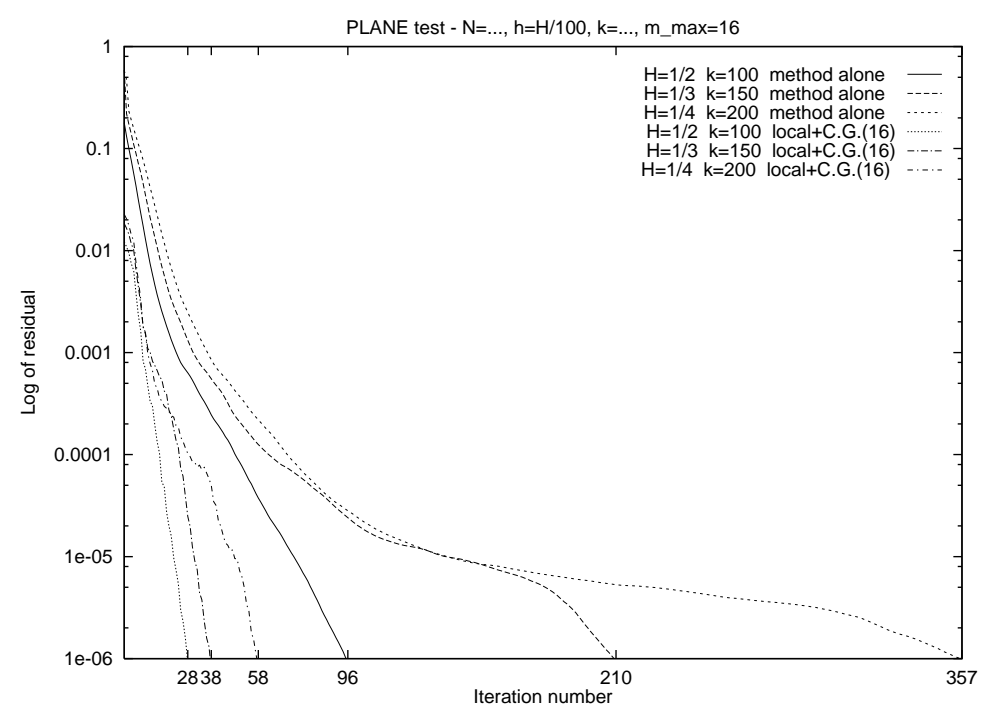

FiguRE 14. Effect of the coarse grid size

preconditioner constructed using a coarsening theory for filtering low frequency errors and accelerating convergence in the presence of fine mesh partitions. A unique characteristic of the proposed method is that, even in the absence of both preconditioners, its number of iterations grows at most polylogarithmically with the number of elements per substructure, and grows sublinearly with the wave number. Furthermore, when equipped with both the local and global preconditioners derived in this paper, the performance of the proposed method becomes almost insensitive to the frequency range and number of subdomains, which makes this method uniquely efficient at solving high frequency exterior acoustics problems [5].

\section{Acknowledgements}

The US authors acknowledge the support by the Office of Naval Research under Grant N-00014-95-1-0663.

\section{References}

1. A. de la Bourdonnaye, Some formulations coupling volumic and integral equation methods for Helmholtz equation and electromagnetism, Numerische Mathematik 69 (1995), 257-268.

2. _ A substructuring method for a harmonic wave propagation problem: Analysis of the condition number of the problem on the interfaces, rapport de recherche No 95-35, CERMICS, France, 1995.

3. B. Desprès, Décomposition de domaine et problème de Helmholtz, Compte Rendu de l'Académie des Sciences No 311 (Série I) (1990), 313-316.

4. B. Desprès, Domain decomposition method and the Helmholtz problem, Mathematical and Numerical aspects of wave propagation phenomena, France, Strasbourg, 1991, pp. 44-52.

5. C. Farhat, P. S. Chen, and J. Mandel, Scalable Lagrange multiplier based domain decomposition method for time-dependent problems, Int. J. Numer. Meth. Engrg. 38 (1995), 3831-3853.

6. C. Farhat, P.-S. Chen, F. Risler, and F.-X. Roux, A simple and unified framework for accelerating the convergence of iterative substructuring methods with Lagrange multipliers, International Journal for Numerical Methods in Engineering, in press.

7. C. Farhat, A. Macedo, and M. Lesoinne, The FETI-H method for the solution of highfrequency exterior Helmholtz problems, submitted to Comput. Meths. Appl. Mech. Engrg. 
8. C. Farhat, A. Macedo, F. Magoulès, and F.-X. Roux, A Lagrange multiplier based domain decomposition method for the exterior Helmholtz problem, Proceedings Fourth U.S. National Congress on Computational Mechanics, San Francisco, California, August 6-8, 1997.

9. C. Farhat and F.-X. Roux, A method of finite element tearing and interconnecting and its parallel solution algorithm, Internat. J. Numer. Meths. Engrg. Vol. 32 (1991), 1205-1227.

10. Charbel Farhat and François-Xavier Roux, Implicit parallel processing in structural mechanics, Computational Mechanics Advances (J. Tinsley Oden, ed.), vol. 2 (1), North-Holland, 1994, pp. 1-124.

11. C. Lacour and Y. Maday, Two different approaches for matching nonconforming grids : the mortar element method and the feti method, B.I.T. (1997).

12. P. Le Tallec, Domain decomposition methods in computational mechanics, Computational Mechanics Advances 2 (1994), 121-220.

13. F.-X. Roux, Méthode de décomposition de domaine pour des problèmes elliptiques, Revue Calculateurs Parallèles, Volume 7, No 1, June 1994.

Cermics, inRIA Sophia Antipolis, France

E-mail address: armel.de_La_bourdonnaye@sophia.inria.fr

University of Colorado,Department of Aerospace Engineering Sciences, Campus Box 429, BOulder CO 80309-0429

E-mail address: charbel@alexandra.Colorado.edu

University of Colorado,Department of Aerospace Engineering Sciences, Campus Box 429, BOulder CO 80309-0429

E-mail address: macedo@alexandra.Colorado.edu

Onera, Direction de l'Informatique, 29 Av. De la Division Leclerc, BP72 92322 Chatillon Cedex, France

E-mail address: magoules@onera.fr

Onera, Direction de l'Informatique, 29 Av. de la Division Leclerc, BP72 92322 Chatillon Cedex, France

E-mail address: roux@onera.fr 